CASE vi. Central Necrosis of Astragalus.-Frances S., aged 12, a delicate looking girl, was admitted on December 18th, 1881. Twelve months before admission, swelling appeared on the outer side of the ankle. Pain, most severe at night, preceded the swelling by some months. Gradually the whole ankle-joint became soft and swollen, like an ordinary white swelling of the ankle. Tenderness on pressure was most marked over the lower end of the fibula. A plaster-of-Paris splint was worn till March 21st, I882, when she returned to the hospital, feeling no better. In less than a month after removing the plaster-ofParis, and whilst the limb was still kept fixed, the ankle-joint showed undoubted signs of suppuration; the temperature rose, and rigors set in. On April 17th, an incision was therefore made in front of the inner, and another behind and below the outer, malleolus. On introducing the finger into the outer wound, I felt a small movable fragment of rough bone close to the articular facet for the outer malleolus. This was lodged in a cavity in the astragalus which was large enough, after the removal of the fragment, to receive the tip of the finger, and was limited by perfectly smooth and uniformly hard walls. The joint was washed out with a solution of tincture of iodine, the wounds covered with terebene and oil on lint, and the limb immovably fixed on a splint. Subsequently, the terebene was changed for iodoform, which was dusted over the weak exuberant granulations which had formed. The limb was still kept immovably fixed. No pain has been suffered since the operation; the joint has resumed almost its natural shape, and the wounds are all but cicatrised. I look forward to the patient recovering with a slightly movable joint.

REMARKS. - The operation which was performed in these cases was not, strictly speaking, trephining. In two of them, after dividing the periosteum by a linear incision, and peeling it off for a certain distance, pus was seen to ooze up through a small opening in the bone; and the abscess-cavity was opened up by applying the gouge to this spot.

In three other cases, after dividing and peeling off the periosteum in the same way, a drill was first introduced, and then the drill-hole was enlarged with the gouge. It is not always sufficient to use a drill under the impression that, if a cavity be reached, pus will escape from it along the side of the drill, or through the hole made by it, after its removal; for, as Case I shows, a little pus with a fragment of necrosed bone may occupy a cavity, but not declare itself by welling up; or the pus may be only semifluid, and too thick to flow, as in Case v. Under either of these conditions, the use of the gouge is needed to expose the abscess.

After emptying it of its contents, the walls of the cavity were in each case well scraped, or rubbed with dry lint, or a fragment of sponge ; it was then washed out with iodine solution; and absorbent cotton-wool, cr lint soaked in terebene and oil (I part to 6), was introduced.

In all the cases the limb was immovably fixed in splints; and the subsequent treatment consisted in irrigating the cavity with iodine solution (tincture of iodine, four drachms to the pint of warm water) daily, or every second or third day, according to the amount of discharge; and the application of the terebene and oil on lint.

The situation of the abscess in Case I was on the epiphysal portion of the humerus, though all the epiphyses had years before become consolidated with the rest of the bone. In the next four cases, one or the other extremity of the diaphysis of a long bone was the seat of disease, though in only one of them was the neighbouring epiphysis consolidated with the shaft. In Case VI, the abscess-cavity was in an irregular, not a long bone. Though this is unusual, it is by no means unique. I have seen the os calcis similarly affected; Mr. Annandale has reported a case in which the lower jaw was the seat of a chronic circumscribed abscess; and most surgeons have seen the mastoid bone similarly affected from extension of disease f:om the middle or external ear.

In the case in which the epiphysal end of the humerus was affected the elbow had become contracted, and the bone between the abscess and the articulating surface was softening down. In all probability, the elbow joint itself would soon have been disorganised. In three cases in which the ends of the diaphyses were affected, the joints were not involved; but in two of them (Cases III and IV) the abscesses, after remaining chronic for some months, took on a more acute form, and led to inflammation of the surrounding soft tissues. In Case IV it produced extensive suppuration in them, and fistulæ were beginning to form ; whilst, in Case III, diffused abscess in the leg would certainly have occurred had the disease not been cut short by operation.

It is interesting to note that in both these cases (Cases III and IV) little or no pain was suffered for a time prior to the soft parts being affected. This was probably owing to the breaking down, in the course of the disease, of the solid inflammatory products which had for a time limited the abscess. . These products, by filling the natural spaces of bone, cause considerable intra-osseous pressure, and excite the severe, dull, and continuous pain which is an usual symptom of chronic abscess in bone.

In Case VI, in which the astragalus was the seat of the disease, the ankle-joint became early involved, and thus in great part, if not en. tirely, the symptoms of circumscribed bone-abscess, or central necrosis, were marked; at any rate, the cause of the suppuration of the anklejoint was not suspected until actually ascertained. It no doubt was an exceptional case, for the great majority of chronic bone-abscesses are in the long bones. But even in them more is seen, in hospital practice, of cases where the abscess has been overlooked or neglected, and has gone on to extensive disorganisation of the bone which it involves, or of the joint in nearest proximity to it, than of cases in which the abscess, still circumscribed within narrow limits, is the only cause of suffering.

To avoid the very serious sequelæ of this affection, as well as to give relief to the distressing pain which it occasions, the diagnosis of boneabscess and central necrosis is a matter of great importance. I make no distinction between the cases in which pus alone, and pus with a small sequestrum, is contained in the cavity, because there is an identity in the processes resulting in the formation of such sequestra, and in those producing small sloughs of connective tissue in the acute circumscribed inflammations of soft parts.

The diagnosis, it must be confessed, is often very difficult : chronic periostitis, chronic osteitis, and cysts and solid tumours of bone, need to be distinguished from abscess in bone. Periosteal thickenings more commonly affect the middle parts of the shafts of long bones, and are generally amenable to local medicinal treatment; whereas chronic abscess in bone generally attacks the ends of the diaphyses or the epiphyses, and is not in the least benefited by remedies.

Cysts in bone will be rightly treated by being laid open in the same way as a chronic abscess; and chronic osteitis, of the form which gives rise to severe continuous or neuralgic pain (the ostéte a forme névralgique of the French), will be relieved or cured by the use of the trephine; whilst the solid tumours require amputation or excision.

Thus, though an accurate diagnosis is admittedly difficult, if not impossible, in some cases, the possibility of falling into error, in spite of careful deliberation, should not discourage us from making an exploratory operation in doubtful cases of disease of bone.

Indeed, I would venture to say that, if we made it a rule to trephine or drill in all cases of chronic enlargement of bone attended with continuous or paroxysmal pain, and which rest and remedies have failed to cure, we should have the satisfaction of saving life itself in some instances, and of restoring to ease and usefulness the limb in very many others.

\section{ON BORO-GLYCERIDE IN OPERATIVE SURGERY.}

BY RICHARD BARWELL, F.R.C.S., Senior Surgeon, Charing Cross Hospital.

Presented to the Section of Surgery, at the Annual Meeting of the British Medical Association in Worcester, Ausust 1882.

THere can be no doubt that the dressing of operation-wounds, by some material, and in some method, that shall prevent fermentation or putrefaction of their secretion, greatly promotes their safety, and is almost a prophylactic against those secondary constitutional effects which we know by the names pyæmia, septicæmia, etc. But there is great doubt as to whether the particular method introduced by Mr. Lister is the best means of obtaining the object in view. It certainly is a very inconvenient one; and, although in hospital practice we may easily, and in the practice of a town, where distances are small, may with some difficulty provide or transport all the cumbersome paraphernalia required, the method can hardly be carried out in the rural practice of a wide district, especially if operation be needed on a sudden emergency. In military surgery, during war time, it is simply an im. possibility.

Time will not allow me to discuss here the probable uselessness of the spray directed upon the wound. Certain is it that the pulverised stream and the eddies it produces cause more atmospheric dust to fall on the bare surface than would otherwise be the case. If we give full credence to the germ-theory, and believe that germination of certain parts of this dust is the direct cause of putrefaction, it must, nevertheless, be acknowledged that, however rapid the reproduction may be, it yet occupies an appreciable time. Therefore if, no spray being used, the wound is immediately washed with the germicide, whatever it may 
be, we can quite as effectually destroy those micrococci that may have been deposited as we can do while yet they are floating in the air.

But carbolic acid is a topical and general poison. ${ }^{*}$ The local irritation is such that the wound has to be guaranteed against its continued contact by a material termed protective; and even then, pretty severe irritation, causing excessive wound-secretion, is the rule rather than the exception; and, when the cancellous bone-structure of young subjects is laid bare, as in excisions, a local osteomyelitis is very apt to be produced.

The absorption of carbolic acid by the wound has been deadly, + but has more frequently been not quite fatal. In the early part of this year (February), I removed a fibroma from a lady's back, under the spray, and used the usual mode of carbolic acid dressing. That night I was sent for, and found my patient apparently sinking. A little urine had been passed about an hour before ; it was quite inky. There was frequent vomiting, and nothing could be retained on the stomach. Pulse 48 ; breathing $3 \mathrm{I}$, very shallow; pupils rather contracted, scarcely responding to light. That this was not the vomiting of ether was shown by the fact that, when I visited her abuut four in the afternoon, she had only vomited once, and had taken some food without difficulty. The operation was at nine in the morning, and the distressing symptoms came on almost exactly twelve hours after. Besides using the spray, the wound had been filled with a 3 per cent. solution of carbolic acid; but, as I had placed a good pad over it, all must I think have been squeezed out.

Impressed by these considerations, but chiefly by the records of carbolic poisoning, I have for some time past been endeavouring to find a reliable safe antiseptic. Thymol, in my hands, entirely failed, nor has the eucalyptus answered my expectations. Boracic acid seemed to me the most reliable substance. I found it, however, very unmanageable, and was actually engaged in trying to overcome certain difficulties, when, on April Ist, I882, Professor Barff's lecture on the preservative qualities of boro-glyceride appeared. Mr. Barff courteously at once complied with my request for a sample; and, after a few experiments, which I may be excused for omitting, I began its use on the human sulject. I have brought some of this substance with me; it is, as will be seen, a soft solid, softer now than in colder weather; by placing the bottle for a while in very hot water, the material comes to be of the consistence of treacle. I do not conceive that the solution of boracic acid in glycerine-for this is merely a saturated solution in the hot fluid-confers upon the former any new quality; it merely renders it more manageable, especially more soluble in water, and more miscible with other substances.

The mode in which I generally use it is as follows. For economy's sake, I generally cleanse my hands and instruments, also the patient's skin, with carbolic acid. I then operate in the open, without any spray or further precaution. The operation completed, the wound is thoroughly mopped and sponged with a five per cent. solution of boroglyceride in water (that is, one ounce to a pint) ; or, in case of a cupshaped wound, I fill it with the solution. After this, the wound is stitched and covered with eight or ten layers of lint, and, where necessary, as in amputations, also with a light bandage similarly soaked. The whole is then enveloped in thin mackintosh; if no oozing take place, there is no need for dressing again for three or four days, sometimes for more. When the dressing is removed, all parts of the wound where the lips have been adjusted are found united or uniting, according to the time elapsed, the surrounding skin being entirely free from redness or any sign of irritation; it is, on the contrary, soft and white like that of a baby. One point in the dressing which I have left till now is the drainage, whether it be desirable or not. My cases, of which I will immediately give a summary, have not enabled me to decide that point with absolute certainty. In two cases in which I omitted that appliance, one had for two days a temperature of $99.5^{\circ}$ to $99.8^{\circ}$; one for three days a temperature of $100.2^{\circ}$ to $101.0^{\circ}$, a mere traumatic, not a septic, temperature, since it began at once, and ended in from forty-eight to seventy-two hours. Others, notably a breast case, with glands extracted from the axilla, making a deep hollow, had no fever at all.

I will now give a table of the cases which I have treated with boro-glyceride after the manner described, omitting three or four minor operations, which all healed with equally remarkable celerity. It is only necessary to premise that no condition can better test the value of an antiseptic than a large deep abscess; the first three cases are of that description.

* Certain experiments of Buchner and others go to show that carbolic acid does not destroy the life of baccilli; it merely renders the wound surface an unfavourable nidus for their reproduction.

† See Case by Mr. Gould, Clinical Society's Transactions, vol. xiii, p. 2or.

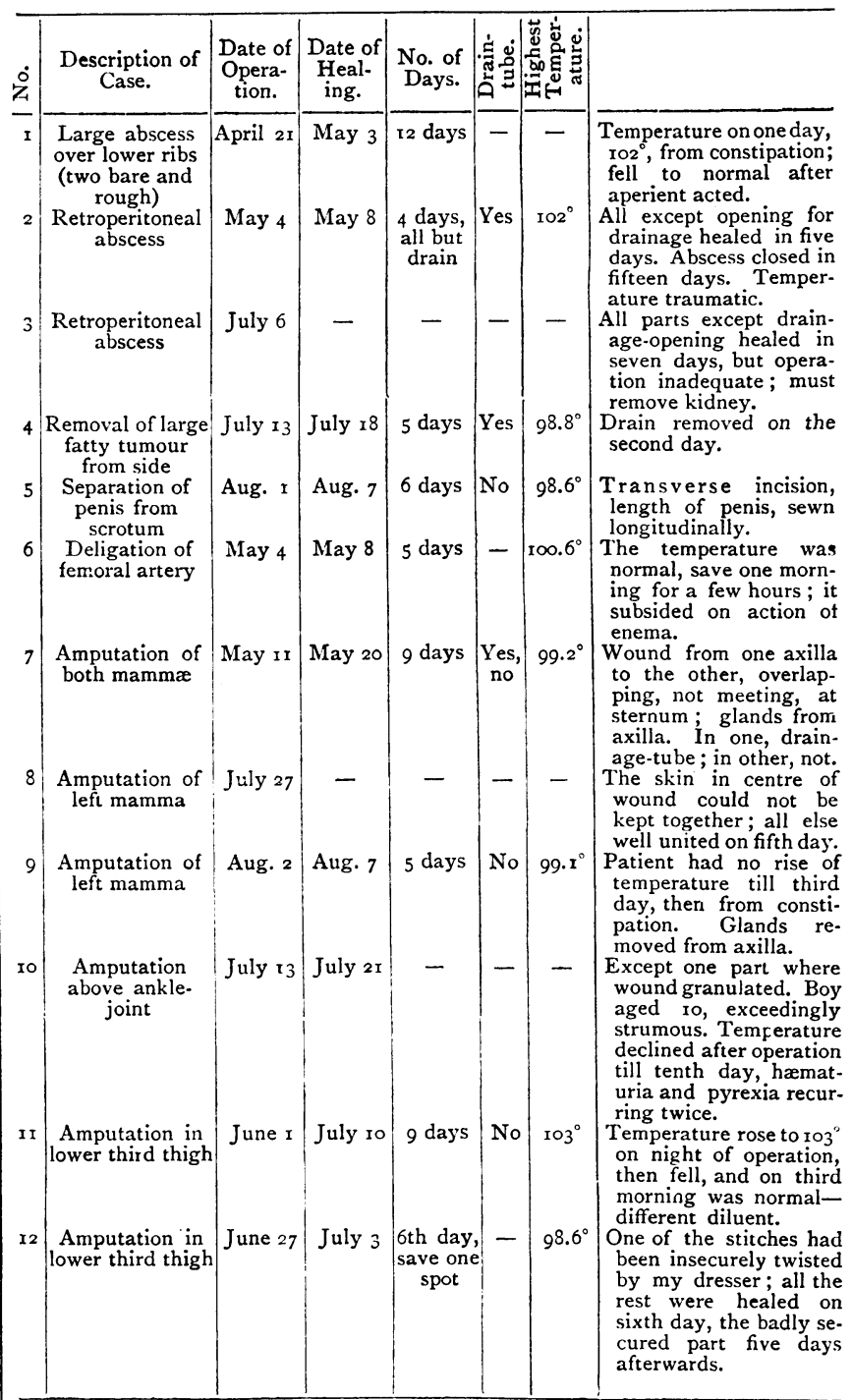

Now these cases, though not very many in number, show clearly that boro-glyceride is not only a perfectly reliable antiseptic, but that it promotes rapid healing of wounds.

Of three amputations of the mamma, one cannot heal quickly, be. cause I was obliged in one part to leave a rather wide gap, but even in these, wherever the lips were in contact, union took place in five days. Of the other two, one was double, and the wourd was enormous, at one place its edges would not be quite adapted, yet I sent the patient back to Mr. Hughes, her attendant at Deal, on the eleventh day; all healed save a small granulating spot. Of the third case, to which I was called by Dr. Farr, of Kensington, I can only say, that the rapidity of healing was almost marvellous. She had no pain nor fever, and sat up for half an hour on the third day.

In one amputation of the thigh, I used as my diluent vaseline; it did not do as well as an aqueous solution. The other amputation of thigh was healed in six days, save one spot where a suture had been insufficiently secured, and the skin therefure allowed to gape. Had it not been for this piece of carelessness, I would have boasted of getting a thigh amputation well within a week; as it is, the man got up on the seventh day. The other amputation healed very well, although such complications as hæmaturia, probably indicating tuberculosis of the kidneys, will keep the lad long under care. The other cases need no comment.

I would point to the fact that the ease and celerity of dressing, and the absence of a spray wetting everything within its radius, are a great gain to the patient as to the surgeon. The simplicity of arrangement, the freedom from cumbersome apparatus, render this method particularly well adapted for military surgery, and for practice in rural districts, 
and I firmly believe it will be found to conduce quite as much as any other method to the absence of blood-poisoning, and more than any other to rapidity of convalescene:

Let me also call attention to the use of this material for injection into the bladder in cystitis. When the mucus and urine are inclined to decompose and to become ammoniacal, this compound acts a charm. I have found it invaluable if any irritation arise after litholapaxy.

\section{ON HYSTERECTOMY.}

Read in the Section of Obstetric Medicine at the Annual Meeting of the British Medical Association in Worcester, August 1882.

BY G. GRANVILLE BANTOCK, M.D.,
Surgeon to the Samaritan Hospital, etc.

THE subject to which I wish to direct your attention to-day is that of Hysterectomy. What I mean by this term is the removal by abdominal section of a fibroid or fibrocystic tumour of the uterus, whether involving a part or the whole of the body of the uterus, or not. It is scarcely necessary, on an occasion like this, that I should defend the nomenclature at any length. I would merely point out that the term hysterectomy, used by some authors, has already been appropriated to Simpson's operation of division of the cervix; that Freund's operation describes the complete extirpation of the uterus, and Porro's the removal of the body of a pregnant uterus, together with both ovaries. Now, the operation of extirpation of a fibroid or fibrocystic tumour varies so much in its details, that it is impossible to find a name, based on anatomical considerations, that will include every case; yet the term hysterectomy appears to me to meet the essentials of the case, and I accept it as sufficiently comprehensive and descriptive. Besides, the best names are not those which most closely and correctly describe any operation, as in two of the instances just mentioned, and in others that will occur to you.

Forty years ago, this operation was unknown. It is true, however, that the abdomen had been opened more than once, viz., even as early as 1825 and 1826 ; but the recognition of the uterine nature of the disease deterred the operators from proceeding further. It was reserved for Dr. Clay of Manchester-whose claims as the pioneer of ovariotomy in this country have, by the way, not been sufficiently recognisea in some quarters-to lead the way in this matter also, by his operation in August 1843. For several years, the operations were "few and far between", and invariably with a fatal result; and it was ten years ere a success was scored. America claims this in the case of Burnham, and, with those of Kimball and Boyd, has the credit of the first three. Nearly twenty years elapsed ere a successful case occurred in this country ; and this, again, falls to the credit of Dr. Clay. Here, however, it is right that I should mention that, in $185 \mathrm{I}, \mathrm{Mr}$. Baker Brown, after removing a multilocular tumour of the right ovary, successfully cut away a small pediculated fibroid, the size of a hen's egg. For the first twenty years, the operations averaged about one a year; and it is a curious fact that these operations, with one exception, were the result of an error of diagnosis ; the operator, in each case, believing he had to deal with an ovarian tumour. According to Péan, the history of this operation comprehends three distinct periods. "In the first, which extends to 1843 , surgeons meeting with fibroid or fibrocystic tumours of the uterus instead of ovarian cysts recoiled before the consequences of amputation of the uterus, and did not complete the operation." In the second, which he calls the period of attempts and gropings, and which comes down to 1863 , "surgeons, emboldened by a more extended practice of ovariotomy, and encouraged by the success obtained in cases complicated by adhesions, which at first it seemed impossible to overcome, took a step in advance, and no longer feared to take away the body of the uterus whenever an error of diagnosis placed them in presence of a tumour in intimate connection with this organ. They were, as it were, compelled to improvise the operation, everything having been disposed for an ordinary ovariotomy. In the third, the operation entered on a new phase. In the month of April 1863, Kœberlé, finding himself in presence of a doubtful tumour, made arrangements for any eventuality; he practised gastrotomy, determined not to recoil even if, in urder to remove the whole of the tumour, he should be obliged to have recourse to supravaginal amputation of the uterus. His courage was rewarded with success."

On this last point, however, there is some conflict; for, whilst Péan claims for Kicberlé the honour of the first operation after a more or less correct diagnosis, others claim this honour for Kimball. Kimball's case occurred in 1853, and therefore ten years anterior to Kœberlé's.

However this may be, it is a fact that the results of the operation for well nigh thirty years were a fearful mortality, and it was not till ten or twelve years ago that this operation can be said to have been placed on a footing at all creditable to surgery; and we must assign the honour of this to the distinguished French surgeon, Péan. From September 1869 to February 1872 , Péan operated nine times, with the very gratifying result of seven recoveries. I know not what Péan's statistics are now, but up to April 1875 they presented a marked contrast to anything done in this country on a large scale to the same date, viz., a mortality of only 29 . I per cent., out of 24 cases-a result which I believe was due to his mode of performing the operation-specifically his treatment of the pedicle. Still the general mortality was very great. In I875, Dr. Samuel Pozzi collected a series of I 19 cases, with a mortality of 64.7 per cent. In 1879 , Dr. Letousey published a further series of 84 cases, with a mortality of 42.8 per cent. And even so recently as two years ago, viz., at the Cambridge meeting of this Asso. ciation, Mr. Spencer Wells stated that he had completed the operation in 34 out of 60 cases, in which he had opened the abdomen to find a fibroid or fibrocystic tumour of the uterus, and of these 18 died, and only 16 recovered, or a mortality of 52.9 per cent. But there were bright spots on this hitherto dark picture, for Keith was, in his quiet way, operating with his usual success; with such success that up the present time he has lost only one out of 12 cases, and in 1879 Dr. Thomas Savage (of Birmingham), published a series of 6 cases with 5 recoveries ; a very encouraging result, though somewhat discounted by a reference, in the same paper, to two cases previously done, with a fatal result.

This is not the occasion, nor will time permit me, to enter on the history, symptoms, diagnosis, progress, and termination of fibroid or fibrocystic tumours of the uterus, or to enter on a comparison of the relative merits of hysterectomy and oöphorectomy so dear to the heart of Mr. Lawson Tait. Perhaps, I ought to apologise to Mr. Tait for associating his name with the term oöphorectomy. I use it for brevity's sake, and must be understood as implying what he prefers to call the "Removal of the Uterine Appendages." Suffice it to say that, while on the one hand, it is allowed that fibroid tumours do not often kill, that they have not that fatal tendency which is so characteristic of the ovarian, on the other hand it is now admitted that there are certain cases, and in not a small proportion, in which this operation affords the only hope of relief from very distressing symptoms, or even of averting death. Such are the cases of true fibro-cyst, of cystiform degeneration of a hard fibroid, of cases in which pain is the predominant symptom, and a few in which hæmorrhage threatens the life of the patient, or this combined with pressure on important viscera. It must be obvious that in most of these cases the removal of the uterine appendages can be of no service.

If any one were asked to name the most characteristic symptom of uterine fibroid, he would probably answer " hæmorrhage." But it is a remarkable fact that in only 4 of the 21 cases in which I have operated was this a prominent symptom at the time of operation. In one case, of which I show you a photograph, menorrhagia had been at one time so severe as to produce very marked anæmia, but the patient had recovered from this condition, and the operation was undertaken for the relief of pain. The explanation of this hæmorrhage will be found in the presence of a large mucous polypus, and the pain was probaibly due to the intestinal adhesions and pressure. In one case the pressure was such as to produce great œdema of the lower extremities, and albuminuria to the extent of one-third, which, with profound anæmia, pre. sented no very inviting prospect to the operator.

One does not usually associate the idea of adhesions with fibroid tumour; yet there were adhesions in no fewer than 9 of my 21 cases. In one, the adhesions were universal; viz., to the parietes, omentum, and intestines, and the intestines were adherent to one another, and to the parietes; in three there were adhesions of the omentum only, in two the adhesions were intestinal only, in two pelvic only, and in one they were omental and intestinal.

Every one who knows anything of fibroid tumours, will agree with me that it is not every case that is suitable for operation-assuming that the symptoms are serious-ard chiefly because of the relations of the growth to the pelvic contents. I may also say that I have myself refused to operate in several cases that seemed most favourable for operation, because the symptoms were not such as to justify me in urging such a proceeding. A tumour which grows out laterally from the uterus, and carries with it the broad ligament, offers insuperable difficulties in the control of hæmorrhage; or when the disease encroaches on, or actually involves, the vaginal portion of the cervix. Such cases, if attended with severe hæmorrhage, must be left to the doubtful benefit of oöphorectomy. Hence there must of necessity be a certain 